\title{
Incidencia De Duolingo En El Desarrollo De Las Habilidades Comunicacionales Verbales Del Idioma Ingles A Nivel De Educación Superior
}

\author{
Armas Pesantez Paul Rolando \\ Magister en lingüística aplicada al aprendizaje del inglés \\ Docente de la Escuela Superior Politécnica de Chimborazo, Ecuador \\ Reinoso Espinosa Ana Gabriela \\ Magister en enseñanza de inglés como idioma extranjero \\ Docente de la Escuela Superior Politécnica de Chimborazo, Ecuador

\section{Macías Silva Evelyn Carolina}

Magister en Pedagogía para la enseñanza del inglés como lengua extranjera Docente de la Escuela Superior Politécnica de Chimborazo, Ecuador

\section{Aquino Rojas Marco Antonio}

Ingeniero en administración de empresas turísticas y hoteleras

Docente de la Escuela Superior Politécnica de Chimborazo, Ecuador

Doi:10.19044/esj.2019.v15n16p29 ～URL:http://dx.doi.org/10.19044/esj.2019.v15n16p29

\section{Resumen}

El objetivo de la investigación fue determinar la incidencia de la aplicación de la plataforma DuoLingo en el desarrollo de las habilidades comunicacionales verbales del idioma inglés, en los estudiantes del cuarto nivel de inglés del Centro de Idiomas de la Escuela Superior Politécnica de Chimborazo, durante el periodo académico marzo - agosto 2017. Para ello, se planteó una investigación con método científico, descriptivo, cuasi experimental, bibliográfica y de campo. La muestra fue intencional y estuvo conformada por veinte y uno (21) estudiantes del paralelo "A" (grupo de control) y 23 estudiantes del paralelo "B" (grupo experimental), quienes fueron evaluados en dos momentos (antes y después). El grupo experimental fue sometido a un plan de intervención durante tres meses, el cual consistió en el desarrollo de un conjunto de actividades que contemplaron: el alcance de lenguaje, precisión, fluidez, coherencia, pronunciación y lenguaje corporal; mientras que el grupo de control aplicó el método tradicional. Para la obtención de la información se aplicó un instrumento validado por pares académico, la misma que fue tabulada, analizada e interpretada y sometida a una prueba estadística, que permitió concluir que DuoLingo incidió 
positivamente en el desarrollo de las habilidades comunicaciones verbales del idioma inglés. Por lo que se recomendó que se haga el uso del mismo.

Palabras Clave: Duolingo, Incidencia, Habilidades comunicacionales verbales, Inglés

\title{
Incidence of Duolingo in the Development of the Verbal Communication Abilities of the English Language at a Higher Education Level
}

\author{
Armas Pesantez Paul Rolando \\ Magister en lingüística aplicada al aprendizaje del inglés \\ Docente de la Escuela Superior Politécnica de Chimborazo, Ecuador \\ Reinoso Espinosa Ana Gabriela \\ Magister en enseñanza de inglés como idioma extranjero \\ Docente de la Escuela Superior Politécnica de Chimborazo, Ecuador

\section{Macías Silva Evelyn Carolina} \\ Magister en Pedagogía para la enseñanza del inglés como lengua extranjera \\ Docente de la Escuela Superior Politécnica de Chimborazo, Ecuador

\section{Aquino Rojas Marco Antonio} \\ Ingeniero en administración de empresas turísticas y hoteleras \\ Docente de la Escuela Superior Politécnica de Chimborazo, Ecuador
}

\begin{abstract}
The objective of the investigation consisted of determining the incidence of the application of the Duolingo platform in the development of the verbal communication abilities of the English language in the students of the fourth English language level of the Language Center of the Escuela Superior Politécnica de Chimborazo during the march-august 2017 academic period . For this a descriptive, quasi experimental, bibliographic and field investigation was stated with the scientific method. The sample was intentional and was made up of twenty one (21) students of parallel "A" (control group) and 23 students of parallel "B" (experimental group) who were evaluated in two moments (before and after). The experimental group was subjected to an intervention plan of three months which consisted of the development of an activity conjunct which viewed the language reach, precision, fluency, coherence, pronunciation and corporal language; the
\end{abstract}


control group, on the other hand, applied the traditional method. For the information obtainment, an instrument validated by academic pairs was applied which was tabulated, analyzed, interpreted and subjected to a statistical test which permitted to conclude that Duolinguo had a positive incidence in the development of verbal communication abilities of the English language. This is why it is recommended to use it.

Keywords: Duolingo, Incidence, verbal communication abilities, English

\section{INTRODUCCIÓN}

La retroalimentación asistida por el docente al estudiante, es una estrategia que debe ser utilizada como parte el desarrollo en las destrezas del idioma inglés. Situación que se complica, por el número de estudiantes que conforman un paralelo. Para ello, es necesario utilizar herramientas tecnológicas que briden el apoyo al docente; entre ellas las plataformas virtuales que se encajen en el modelo computacional uno a uno con el fin de que el estudiante pueda construir su propio conocimiento y mediante la plataforma sin horarios restringidos este en constante aprendizaje.

A nivel mundial el idioma inglés ocupa el tercer lugar entre los idiomas más hablados y es empleado en todas las áreas del conocimiento y se ha convertido en un instrumento para la comunicación con personas de diferentes países; por lo que existe la necesidad en la enseñanza de impartirlo utilizando diferentes estrategias, métodos e instrumentos, de manera que los estudiantes puedan adquirirlos motivando su creatividad, dinamismo y diversión; dejando de lado la antigua educación tradicional y poco participativa.

Una de las estrategias ha sido el uso e implementación de ambientes educativos virtuales conocido como plataformas que contienen recursos y actividades dinámicas e interactivas que ayudan al proceso de enseñanza aprendizaje del idioma inglés.

Díaz (1986) sostiene que "Llamamos aprendizaje a la modificación relativamente permanente en la disposición o en la capacidad del hombre, ocurrida como resultado de su actividad y que no puede atribuirse simplemente al proceso de crecimiento y maduración o a causas tales como enfermedad o mutaciones genéticas" (p. 40). La teoría del aprendizaje de un primer o segundo idioma como el inglés consiste cuando ocurre la comprensión de un mensaje real y cuando el receptor puede decodificarlo con éxito.

Alzate (2015) indica que "Se puede inferir que la herramienta Duolingo, se constituyó en un eficaz soporte para mejorar el nivel de aprendizaje del idioma inglés en los estudiantes de grado" (p. 62). Por lo que, 
a través de la investigación se pretende experimentar con una diferente muestra a fin de comprobar si se presenta un resultado similar.

Duolingo es un programa de enseñanza de idiomas interactivo que le permite al estudiante aprender a su propio ritmo. Está basado en la web y guía al estudiante paso a paso a través de una secuencia de tareas basada en la traducción. El sitio web Duolingo (http://www.duolingo.com) afirma que Duolingo está científicamente probado, pues un estudio independiente encontró que esta plataforma triunfa en el aprendizaje de idiomas a nivel universitario.

Gavarri (2016) argumenta que "La motivación y el incentivo que el alumno recibe a través de esta plataforma de aprendizaje se consideran relevantes para explicar algunos de los motivos por los cuales tanta cantidad de usuarios disfrutan del sistema de enseñanza-aprendizaje ofrecido por esta plataforma." (p. 9). Vesselinov y Grego (2012) en su investigación "Duolingo Effectiveness Study" financiada por Duolingo, demostraron avances significativos del 91,4\% en las destrezas que implica el aprendizaje del idioma español para estudiantes cuya lengua materna es el inglés; mediante evaluaciones realizadas en una fase inicial y final. La medida de efectividad alcanzó 8,1 promedio por cada hora de estudio en Duolingo, resaltando que la motivación personal de ciertos participantes incidió en el factor de efectividad.

Navarro (2004) afirma que el aprendizaje es un proceso extremadamente complejo de la naturaleza, su punto de partida es la adquisición de nuevo conocimiento, capacidad o destreza. El aprendizaje debe manifestarse en el futuro y contribuir a la solución de problemas concretos, incluyendo diferentes en su origen que motivaron el desarrollo del conocimiento (Navarro \& Buenos Aires. Sede Regional, 2004).

Baddeley (1990) concluye que la motivación condiciona la cantidad de tiempo invertido en el aprendizaje, es decir si esta motivación ínsita de forma positiva en el estudiante el tiempo que le tomará aprender será menos y si no se le proporciona una motivación adecuada el tiempo de aprendizaje se extenderá.

Bóer (1970) y Bransford (1979) demostraron que la organización, e integración de la información facilita la retención y su recuperación en forma de esquema y redes de ideas. Así también Ebbinghaus (1967) ha demostrado que la facilidad de reproducir los recuerdos es directamente proporcional a la fuerza con que los mismos están grabados en la mente del humano, y que su memorización es mejor a través de la repetición sistemática.

En el aprendizaje del idioma inglés, si a mayor tiempo invertido en aprendizaje, mayor será la cantidad de información que el estudiante obtendrá y retendrá en la mente. Aprender de forma mecánica las palabras, reglas gramaticales o frases no es garantía de que como estudiantes aprendamos, sino 
que esta información debe ser analizada y utilizada acorde a la circunstancia en el que el estudiante se encuentre.

Ruiz (2018) “... En un contexto formal de adquisición, se debe incidir más en aspectos tales como la intensidad en el uso del inglés como lengua vehicular y el enfoque pedagógico desarrollado en el aula, a la hora de adquirir una competencia comunicativa escrita mayor." (p. 418).

Canale y Swain (1980) proponen un método de enseñanza comunicativa que destaque el uso de la lengua en situaciones comunicativas significativas, más que un enfoque fundamentalmente gramatical. Dicho método incluye cinco aspectos que a su consideración debe incluir cualquier método de enseñanza comunicativa: competencia estratégica, competencia gramatical, interacción significativa, reglas básicas del discurso, aspectos sociales y cultura de la lengua meta. En resumen, la teoría propuesta por estos autores para la competencia comunicativa considera tres competencias principales: la gramatical, la sociolingüística y la estratégica.

Por lo antes mencionado, en la presente investigación se aplicó un plan de intervención que contiene método, técnica, instrumento y análisis de la información para determinar la incidencia en la habilidad comunicacional verbal.

\section{METODOLOGÍA}

La investigación fue dirigida al proceso pedagógico de enseñanza del idioma inglés, fue de tipo explicativa ya que se analizaron los resultados de investigación y su aplicación.

La investigación fue cuasi experimental, caracterizado por un estudio que determinó la incidencia de la variable independiente (Duolingo) sobre la dependiente (habilidades comunicativas verbales en inglés).

Por el hecho de interactuar y obtener la información de los actores directos de la investigación, se consideró una investigación de campo. Por la fundamentación en fuentes primarias y secundarias de las variables de la investigación, mediante la revisión de la literatura hace que la investigación sea bibliográfica.

La población está conformada por 50 estudiantes entre hombres y mujeres del cuarto nivel de inglés del Centro de Idiomas de la Escuela Superior Politécnica de Chimborazo (ESPOCH). La muestra representa la totalidad de la población dividida en dos grupos: grupo de control paralelo "A" y grupo experimental paralelo "B", durante el periodo académico marzo - agosto 2017; de los cuales 6 desertaron. Por lo que, la muestra final fue de 44 estudiantes, de los cuales son afines a edades entre 20 y 23 años y tienen conocimientos homogéneos de estructura gramatical. Los dos grupos siguen un mismo plan curricular (contenidos similares) pero el grupo experimental fue sometido a un plan de intervención; mientras que el grupo de control no. 
Tabla 1. Muestra intencional

\begin{tabular}{|c|c|c|c|}
\hline & GRUPO CONTROL & $\begin{array}{c}\text { GRUPO } \\
\text { EXPERIMENTAL }\end{array}$ & Total \\
\hline $\begin{array}{c}\text { Estudiantes del } \\
\text { cuarto nivel de } \\
\text { inglés }\end{array}$ & $\begin{array}{c}21 \\
\text { Paralelo "A" }\end{array}$ & $\begin{array}{c}23 \\
\text { Paralelo "B" }\end{array}$ & $\mathbf{4 4}$ \\
\hline Total & $\mathbf{2 1}$ & $\mathbf{2 3}$ & $\mathbf{4 4}$ \\
\hline
\end{tabular}

Fuente: Sistema académico ESPOCH

Elaborado por: Armas Paúl, 2017.

El plan de intervención se desarrolló durante de 20 semanas, basados en el modelo computacional uno a uno, es decir que la máquina enseña constantemente y se adapta al estudiante a través de retroalimentación (feedback).

La variable independiente para la investigación fue Duolingo, que es una herramienta tecnológica de aprendizaje computacional uno a uno, que fue dimensionada por actividades que implican el desarrolla la habilidad computacional verbal, que son dirigidas por el docente y que forman parte del plan de intervención; mientras que la variable dependiente representa las habilidades comunicacionales verbales que son consideradas como un proceso a ser desarrolladas mediante la incidencia del alcance de lenguaje, precisión, fluidez, coherencia, pronunciación, lenguaje corporal.

La investigación inició con la evaluación, mediante un test inicial (pre test) que determinó el nivel de conocimientos tanto del grupo de control como al grupo experimental, detectando las falencias a ser fortalecidas mediante el plan de intervención, y en los casos necesarios aplicar clases guiadas y autónomas a través de la plataforma Duolingo, la cual permite realizar dichas actividades.

El plan de intervención, trata los contenidos del plan analítico, mediante clases dirigidas que contempla un conjunto de actividades que inducen el desarrollo las habilidades comunicativas verbales de inglés enmarcadas en los modelos pedagógicos conectivista y conceptual.

Para la evaluación de los docentes hacia los estudiantes, se utilizó la técnica de la observación y se puso a disposición una lista de cotejo, que fue revisada y verificada por pares académicos y que alcanzó un coeficiente aceptable de confiabilidad de 0,8105 según Alfa de Cronbach. La rúbrica permitió evaluar seis (6) descriptores entre ellos: el alcance de la lengua en general, a través de un repertorio de palabras frases de comunicación efectiva. La precisión evaluó el uso correcto de la gramática comunicacional. La fluidez estableció la expresión sin pausas o dudas. La coherencia del discurso y uso de conectores. La pronunciación de entonación apropiada y articulación correcta de sonidos ingleses y finalmente el lenguaje corporal del uso no verbal o gestual para captar la atención de la audiencia. 
La rúbrica consta de opciones que el docente debe ir seleccionado según el estudiante va desarrollando su participación. Para desarrollar las actividades con el grupo experimental, se cuenta con un laboratorio de 30 computadoras provistas de internet inalámbrico y por cable.

Fue necesario una breve guía del uso de la plataforma Doulingo por parte del docente, para posteriormente en las restantes semanas aplicar el plan de intervención que incluye la recolección de los resultados.

Tanto el grupo de control como el grupo experimental fueron evaluados antes, durante el proceso de enseñanza, obteniendo varias evaluaciones, las mismas que fueron cuantificadas, tabuladas analizar e interpretadas. Los resultados obtenidos obtenidas del pre test y post test de cada grupo fueron filtradas, revisadas para evitar errores, tabuladas, representadas mediante tablas y gráficos; para posteriormente aplicar una prueba estadística en las que los resultados fueron comparados y tomados como referencia para establecer si la hipótesis de la investigación fue cumplida.

\section{RESULTADOS}

A continuación, se presenta los resultados de las evaluaciones obtenidas de los veintiuno (21) estudiantes del grupo de control y veinte y tres (23) estudiantes del grupo experimental del cuarto nivel del Centro de Idiomas. Para ello se consideró la rúbrica con los descriptores con opciones que fueron ponderadas de la siguiente manera:

Tabla 4. Ponderación de opciones de rubrica

\begin{tabular}{|c|c|c|c|c|}
\hline \multicolumn{5}{|c|}{ Descriptor: Alcance de lenguaje } \\
\hline Excelente & Muy bueno & Bueno & Regular & Deficiente \\
\hline 1,5 & 1,2 & 0,9 & 0,6 & 0,3 \\
\hline \multicolumn{5}{|c|}{ Descriptor: Precisión } \\
\hline Excelente & Muy bueno & Bueno & Regular & Deficiente \\
\hline 2,0 & 1,6 & 1,2 & 0,8 & 0,4 \\
\hline \multicolumn{5}{|c|}{ Descriptor: Fluidez } \\
\hline Excelente & Muy bueno & Bueno & Regular & Deficiente \\
\hline 2,0 & 1,6 & 1,2 & 0,8 & 0,4 \\
\hline \multicolumn{5}{|c|}{ Descriptor: Coherencia } \\
\hline Excelente & Muy bueno & Bueno & Regular & Deficiente \\
\hline 1,0 & 0,8 & 0,6 & 0,4 & 0,2 \\
\hline \multicolumn{5}{|c|}{ Descriptor: Pronunciación } \\
\hline Excelente & Muy bueno & Bueno & Regular & Deficiente \\
\hline 2,0 & 1,6 & 1,2 & 0,8 & 0,4 \\
\hline \multicolumn{5}{|c|}{ Descriptor: Lenguaje corporal } \\
\hline Excelente & Muy bueno & Bueno & Regular & Deficiente \\
\hline 1,5 & 1,2 & 0,9 & 0,6 & 0,3 \\
\hline \multicolumn{2}{|l|}{ Descriptor } & \multicolumn{2}{|c|}{ Puntaje } & Total puntaje \\
\hline \multicolumn{2}{|c|}{ Alcance de lenguaje } & \multicolumn{2}{|c|}{1,5} & \multirow{4}{*}{10} \\
\hline \multicolumn{2}{|l|}{ Precisión } & \multicolumn{2}{|c|}{2,0} & \\
\hline \multicolumn{2}{|l|}{ Fluidez } & \multicolumn{2}{|c|}{2,0} & \\
\hline \multicolumn{2}{|l|}{ Coherencia } & \multicolumn{2}{|c|}{1,0} & \\
\hline
\end{tabular}




\begin{tabular}{|l|l|l|}
\hline Pronunciación & 2,0 & \\
\hline Lenguaje corporal & 1,5 & \\
\hline
\end{tabular}

Elaborado por: Armas Paul, 2017

Obtenidas las evaluaciones de los estudiantes se analizan los resultados a través de la siguiente gráfica

\section{Calificaciones del Pre test (Grupo control vs Grupo Experimental)}

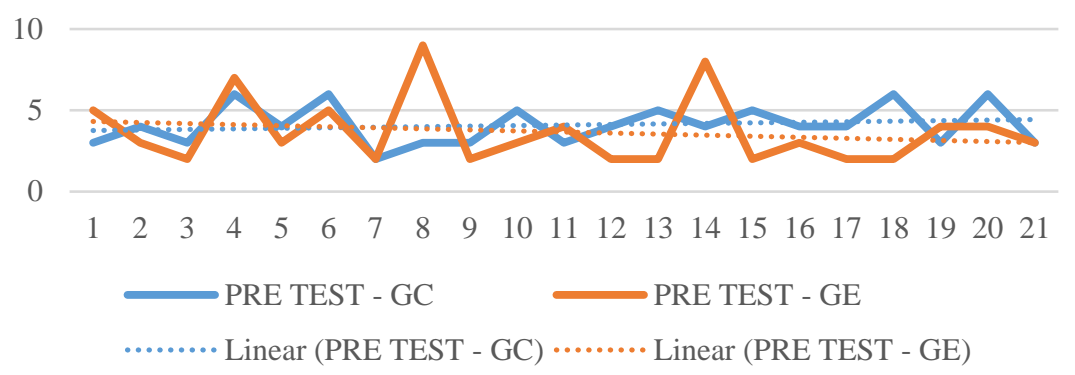

Gráfico 3. Calificaciones del Pre test (Grupo control vs Grupo Experimental)

Al observar el gráfico 3, se puede evidenciar que en promedio los estudiantes del grupo de control obtuvieron mejores calificaciones (/10 puntos) que los estudiantes del grupo experimental; con respecto a los descriptores que evaluaron las habilidades comunicativas verbales.

Según las calificaciones obtenidas, se pudo observar que el grupo experimental es heterogéneo, ya que existen estudiantes con notas altas y otros con notas bajas; mientras que el grupo de control es homogéneo,

Se puede argumentar que, en ambos grupos en promedio, requieren desarrollar las habilidades comunicaciones verbales del idioma inglés; es decir que es un escenario ideal para aplicar el plan de intervención a través de la plataforma Duolingo.

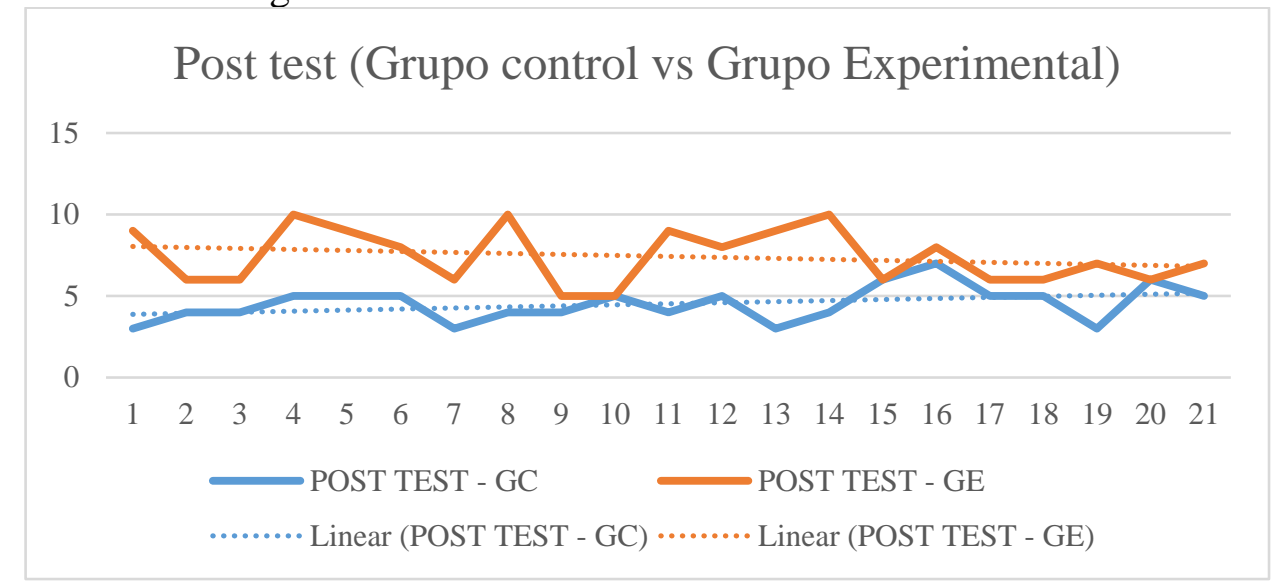

Gráfico 4. Post test (Grupo control vs Grupo Experimental) 
Es notorio en el gráfico 4 que, en la evaluación del post test el promedio de las calificaciones de los estudiantes del grupo experimental, superan a los estudiantes del grupo de control; así lo muestra la línea de tendencia del grupo experimental. Estos resultados son obtenidos una vez que se ha realizado la intervención con la plataforma Duolingo, para desarrollar las habilidades comunicacionales verbales.

Por otra parte, el grupo de control mantiene sus calificaciones con respecto al pre test, y muestra un ligero incremento en las calificaciones. Considerando que existieron estudiantes que sí lograron alcanzar evaluaciones de hasta 7 puntos; como también existieron estudiantes del grupo experimental que a pesar de la intervención no pudieron superar las calificaciones obtenidas en el pre test.

Sin duda, el modelo computacional uno a uno concebido en la plataforma Duolingo, permitió que el estudiante de forma autónoma aprenda y se retroalimente de forma continua en los temas revisados en clase. Es decir que, mediante las actividades se logró que las evaluaciones en los descriptores mejoren en el post test.

A continuación, se presentan los resultados obtenidos de la rúbrica aplicada a los grupos de control y experimental en el pre test y post test

Tabla 5. Frecuencias del pre test y post test del Grupo de Control

\begin{tabular}{|c|c|c|c|c|c|c|c|c|c|c|c|c|}
\hline \multirow[b]{2}{*}{$\begin{array}{c}\text { DESCRIPTOR } \\
\text { ES }\end{array}$} & \multicolumn{2}{|c|}{ Excelente } & \multicolumn{2}{|c|}{$\begin{array}{c}\text { Muy } \\
\text { bueno }\end{array}$} & \multicolumn{2}{|c|}{ Bueno } & \multicolumn{2}{|c|}{$\begin{array}{c}\text { Regula } \\
\mathbf{r}\end{array}$} & \multicolumn{2}{|c|}{ Deficiente } & \multirow[b]{2}{*}{$\begin{array}{c}\text { Total } \\
\text { Pre } \\
\text { Test }\end{array}$} & \multirow[b]{2}{*}{$\begin{array}{c}\text { Total } \\
\text { Post } \\
\text { test }\end{array}$} \\
\hline & $\begin{array}{c}\text { PR } \\
\text { E } \\
\text { TE } \\
\text { ST }\end{array}$ & $\begin{array}{c}\text { POS } \\
\text { T } \\
\text { TES } \\
\text { T }\end{array}$ & $\begin{array}{c}\text { PR } \\
\text { E } \\
\text { TE } \\
\text { ST }\end{array}$ & $\begin{array}{l}\text { PO } \\
\text { ST } \\
\text { TE } \\
\text { ST }\end{array}$ & $\begin{array}{c}\mathbf{P} \\
\mathbf{R} \\
\mathbf{E} \\
\mathbf{T} \\
\mathbf{E S} \\
\mathbf{T}\end{array}$ & $\begin{array}{l}\text { PO } \\
\text { ST } \\
\text { TE } \\
\text { ST }\end{array}$ & $\begin{array}{c}\mathbf{P} \\
\mathbf{R} \\
\mathbf{E} \\
\mathbf{T} \\
\mathbf{E S} \\
\mathbf{T}\end{array}$ & $\begin{array}{l}\text { PO } \\
\text { ST } \\
\text { TE } \\
\text { ST }\end{array}$ & $\begin{array}{c}\text { PR } \\
\text { E } \\
\text { TES } \\
\text { T }\end{array}$ & $\begin{array}{c}\text { POS } \\
\text { T } \\
\text { TES } \\
\text { T }\end{array}$ & & \\
\hline $\begin{array}{l}\text { Alcance de } \\
\text { lenguaje }\end{array}$ & 1 & 2 & 2 & 1 & 6 & 6 & 4 & 5 & 8 & 7 & 21 & 21 \\
\hline Precisión & 0 & 1 & 1 & 2 & 4 & 4 & 6 & 8 & 10 & 6 & 21 & 21 \\
\hline Fluidez & 1 & 1 & 1 & 2 & 3 & 4 & 7 & 6 & 9 & 8 & 21 & 21 \\
\hline Coherencia & 2 & 2 & 2 & 3 & 3 & 2 & 4 & 6 & 10 & 8 & 21 & 21 \\
\hline Pronunciación & 3 & 4 & 2 & 3 & 3 & 5 & 5 & 3 & 8 & 6 & 21 & 21 \\
\hline $\begin{array}{l}\text { Lenguaje } \\
\text { Corporal }\end{array}$ & 2 & 2 & 3 & 4 & 3 & 4 & 4 & 5 & 9 & 6 & 21 & 21 \\
\hline
\end{tabular}

Fuente: Lista de cotejo

Elaborado por: Armas Paúl, 2017 


\section{Frecuencias Pre -Test y Post -Test del Grupo control}

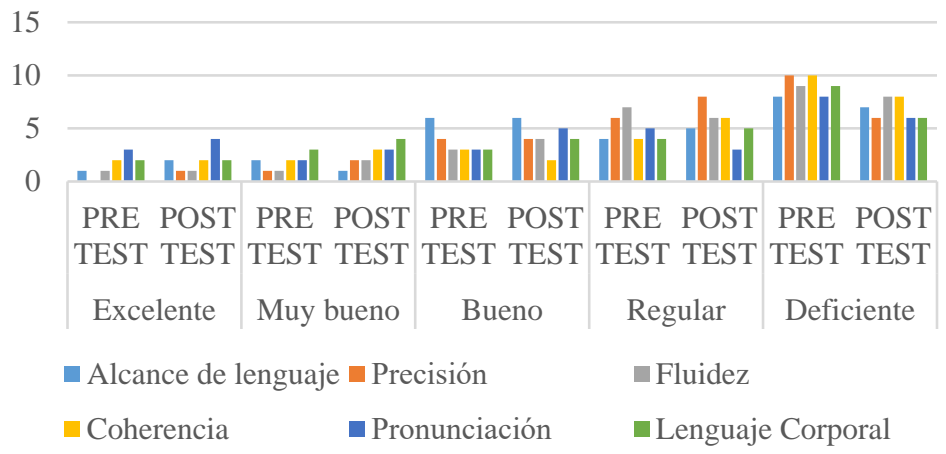

Grafico 5. Barras del pre test y post test del, Grupo de Control

El gráfico 5, estableció que el mayor número de frecuencias tanto en el pre test y post test del grupo de control de los descriptores, tienen mayor tendencia en las opciones de Deficiente y Regular, considerando que al grupo de control no se le aplicado el plan de intervención.

En la opción Bueno los descriptores de la precisión, fluidez, coherencia, lenguaje y corporal, presentan un pequeño incremento; mientras que el alcance del lenguaje se decremento en el post test.

Por otro lado, en las opciones Muy bueno y Excelente las frecuencias no presentan cambios significativos. Es decir, que la tendencia general de las frecuencias, se inclinan a las opciones negativamente en el grupo de control; concluyendo que no existe novedades favorables, en cuanto al desarrollo de las competencias verbales.

Tabla 6. Frecuencias del pre test y post test del Grupo Experimental

\begin{tabular}{|c|c|c|c|c|c|c|c|c|c|c|c|c|}
\hline \multirow[b]{2}{*}{$\begin{array}{c}\text { DESCRIPTORE } \\
\mathrm{S}\end{array}$} & \multicolumn{2}{|c|}{ Excelente } & \multicolumn{2}{|c|}{$\begin{array}{c}\text { Muy } \\
\text { bueno }\end{array}$} & \multicolumn{2}{|c|}{ Bueno } & \multicolumn{2}{|c|}{ Regular } & \multicolumn{2}{|c|}{ Deficiente } & \multirow[b]{2}{*}{$\begin{array}{c}\text { Total } \\
\text { Pre } \\
\text { Test }\end{array}$} & \multirow[b]{2}{*}{$\begin{array}{c}\text { Total } \\
\text { Post } \\
\text { test }\end{array}$} \\
\hline & $\begin{array}{c}\text { PR } \\
\text { E } \\
\text { TE } \\
\text { ST }\end{array}$ & $\begin{array}{c}\text { POS } \\
\text { T } \\
\text { TES } \\
\text { T }\end{array}$ & $\begin{array}{c}\text { PR } \\
\text { E } \\
\text { TE } \\
\text { ST }\end{array}$ & $\begin{array}{l}\text { PO } \\
\text { ST } \\
\text { TE } \\
\text { ST }\end{array}$ & $\begin{array}{c}\text { PR } \\
\text { E } \\
\text { TE } \\
\text { ST }\end{array}$ & $\begin{array}{l}\text { PO } \\
\text { ST } \\
\text { TE } \\
\text { ST }\end{array}$ & $\begin{array}{c}\text { PR } \\
\text { E } \\
\text { TE } \\
\text { ST }\end{array}$ & $\begin{array}{l}\text { PO } \\
\text { ST } \\
\text { TE } \\
\text { ST }\end{array}$ & $\begin{array}{c}\text { PRE } \\
\text { TES } \\
\text { T }\end{array}$ & $\begin{array}{c}\text { POS } \\
\text { T } \\
\text { TES } \\
\text { T }\end{array}$ & & \\
\hline $\begin{array}{c}\text { Alcance de } \\
\text { lenguaje }\end{array}$ & 2 & 6 & 1 & 8 & 5 & 6 & 5 & 1 & 10 & 2 & 23 & 23 \\
\hline Precisión & 1 & 6 & 2 & 7 & 5 & 7 & 7 & 2 & 8 & 1 & 23 & 23 \\
\hline Fluidez & 1 & 5 & 2 & 9 & 4 & 7 & 7 & 1 & 9 & 1 & 23 & 23 \\
\hline Coherencia & 1 & 6 & 1 & 5 & 1 & 6 & 7 & 4 & 13 & 2 & 23 & 23 \\
\hline Pronunciación & 2 & 8 & 1 & 7 & 4 & 3 & 5 & 3 & 11 & 2 & 23 & 23 \\
\hline $\begin{array}{l}\text { Lenguaje } \\
\text { Corporal }\end{array}$ & 2 & 9 & 2 & 8 & 3 & 4 & 6 & 2 & 10 & 0 & 23 & 23 \\
\hline
\end{tabular}

Fuente: Lista de cotejo

Elaborado por: Armas Paúl, 2017 


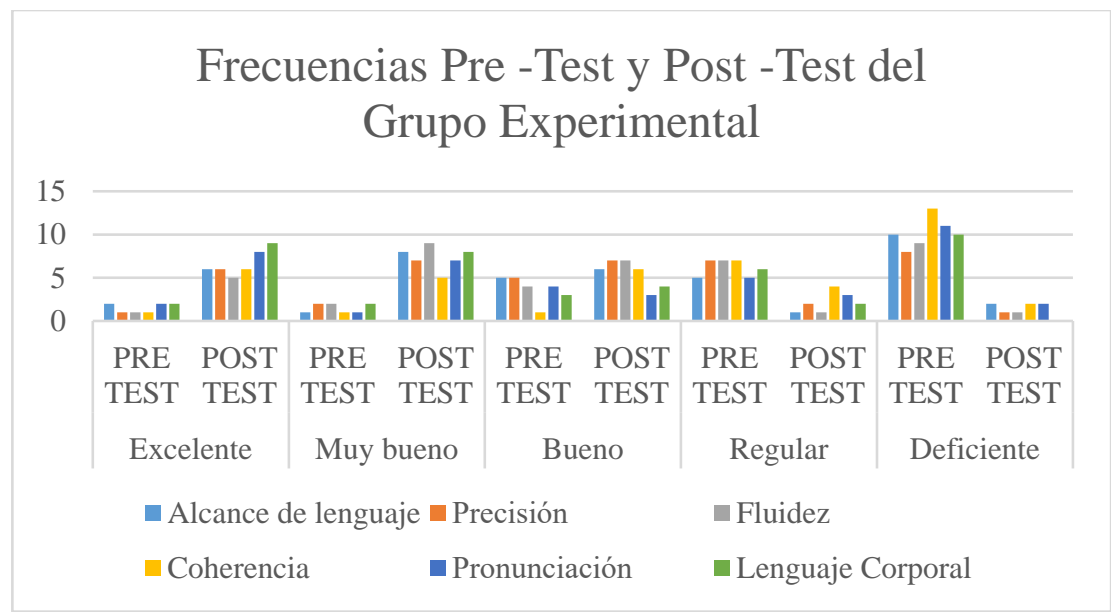

Grafico 6. Barras del pre test y post test de, Grupo Experimental

El gráfico 6, muestra a diferencia del grupo de control que en el pre test el mayor número de frecuencias de los descriptores, se registraron en las opciones de Regular y Deficiente, y una vez aplicado el plan de intervención se revierten a las opciones de Excelente y Muy bueno; mientras que la opción Regular mantiene el registro de frecuencias.

El descriptor que debe ser atendido o debe ser fortalecido, es la Coherencia; a pesar que se encuentra presente en las opciones favorables de las habilidades comunicacionales verbales, luego de aplicar como instrumento de enseñanza a Duolingo.

Al observar el gráfico las opciones que muestran claramente incremento de frecuencias en el post test son las opciones: Excelente y Muy buena, sobre manera el descriptor de lenguaje corporal y la pronunciación

En general las frecuencias claramente muestran que con el plan de intervención y con la ayuda de la plataforma Duolingo se desarrollaron positivamente las habilidades comunicacionales verbales en el grupo experimental.

\section{Análisis e interpretación estadística de resultados}

Tabla 7. Nivel de Confianza - Pre test Grupo de control

\begin{tabular}{|c|ll|}
\hline$\sum \mathrm{f}_{\mathrm{i}}=21 \quad \sum \mathrm{x}_{\mathrm{i}} \mathrm{f}_{\mathrm{i}}=86 \quad \sum \mathrm{f}_{\mathrm{i}} \mathrm{x}_{\mathrm{i}}^{2}=382$ & Media: & 4,095238095 \\
& Desv. Estandar: & 1,19 \\
$\sigma_{c(\text { pre })}=\sqrt{\frac{\sum f X_{i}^{2}}{n_{c}}}-\bar{X}_{c}^{2}$ & Confianza: & $95 \%$ \\
$\mathrm{X}=4,095238095$ & $\mathrm{~N}$. Casos: & 21 \\
$\mathrm{O}:$ & alfa: & 2,56 \\
$\mathrm{C}=1,19$ & Intervalo & $5 \%$ \\
\hline
\end{tabular}

Fuente: Microsoft Excel

Elaborado por: Armas Paul, 2017 
El puntaje medio del grupo control en el pre-test fue de 4,095238095, con una desviación estándar de 1,19. En un intervalo de confianza del 95\% $(z=2,56)$ implica que el $95 \%$ de los puntajes del grupo se encuentra entre 3,59 y 4,60

Tabla 8. Nivel de Confianza - Pre test Grupo de control

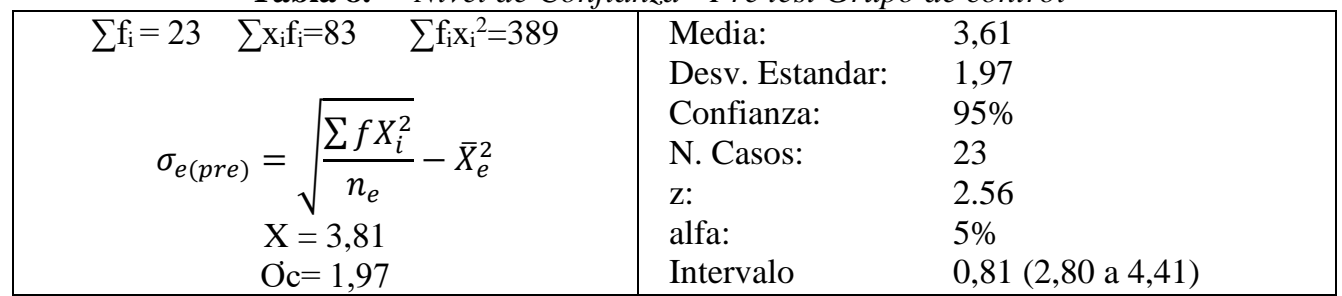

Fuente: Microsoft Excel

Elaborado por: Armas Paul, 2017

El puntaje medio del grupo experimental en el pre-test fue de 3,61 con una desviación estándar de 1,97. En un intervalo de confianza del 95\% $(z=2.56)$ implica que el $95 \%$ de los puntajes del grupo se encuentra entre 2,80 a 4,41

Tabla 9. Nivel de Confianza-Post test Grupo de Control

\begin{tabular}{|c|ll|}
\hline$\sum \mathrm{f}_{\mathrm{i}}=21 \quad \sum \mathrm{x}_{\mathrm{i}} \mathrm{f}_{\mathrm{i}}=95 \quad \sum \mathrm{f}_{\mathrm{i} \mathrm{x}_{\mathrm{i}}{ }^{2}=435}$ & Media: & 4,52 \\
& Desv. Estandar: & 1,05 \\
$\sigma_{c(\text { post })}=\sqrt{\frac{\sum f X_{i}^{2}}{n_{c}}}-\bar{X}_{c}^{2}$ & Confianza: & $95 \%$ \\
$\mathrm{X}=4,523809524$ & $\mathrm{~N}$. Casos: & 21 \\
$\mathrm{O} \mathrm{c}=1,05$ & $\mathrm{z}:$ & 2.56 \\
& alfa: & $5 \%$ \\
\end{tabular}

Fuente: Microsoft Excel

Elaborado por: Armas Paul, 2017

El puntaje medio del grupo control en el post-test es de 4,52 con una desviación estándar de 1,05 traducido en un intervalo de confianza del 95\% $(z=2,56)$ implica que el $95 \%$ de los puntajes del grupo se encuentra entre 4,07 a 4,97 .

Tabla 10. Nivel de Confianza - Post test Grupo Experimental

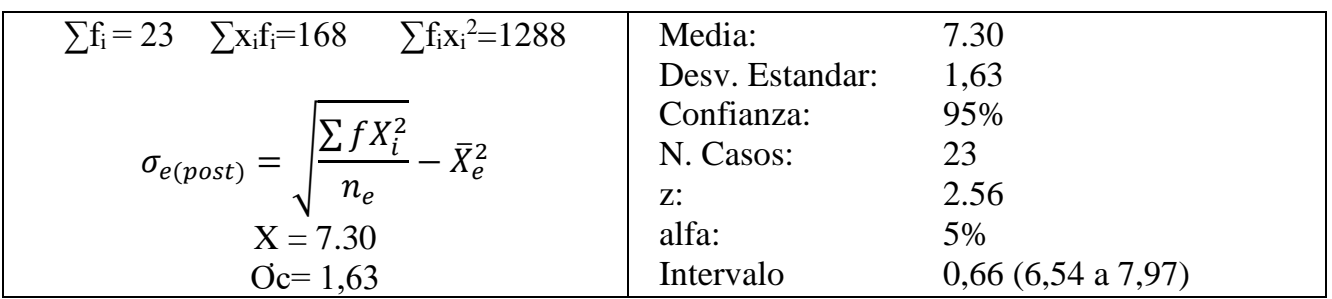

Fuente: Microsoft Excel

Elaborado por: Armas Paul, 2017 
El puntaje medio del grupo experimental post-test es de 7.30 con una desviación estándar de 1,63 traducido en un intervalo de confianza del 95\% $(z=2,56)$, implica que el $95 \%$ de los puntajes del grupo se encuentra entre 6,64 a 7,97 .

\section{Análisis y prueba de hipótesis}

$\mathbf{H}_{0}$ : Las actividades de la plataforma Duolingo no incide en el desarrollo de las habilidades comunicacionales verbales del idioma inglés.

$\mathbf{H}_{1}$ : Las actividades de la plataforma Duolingo incide en el desarrollo de las habilidades comunicacionales verbales del idioma inglés.

\section{Lenguaje matemático}

$$
\begin{gathered}
\text { Hi: } \bar{x}_{e} \neq \bar{x}_{C}: \text { con A1: } \bar{x}_{e}>\bar{x}_{C} \text { o A2: } \bar{x}_{e}<\bar{x}_{C} \\
\text { Ho: } \bar{x}_{e}=\bar{x}_{C}
\end{gathered}
$$

Tabla 11. Análisis y prueba de hipótesis: grupo control

\begin{tabular}{|c|c|c|}
\hline RÚBRICA & $\begin{array}{c}\text { MEDIA } \\
\text { ARITMÉTICA }\end{array}$ & $\begin{array}{c}\text { DESVIACIÓN } \\
\text { ESTÁNDAR(S) }\end{array}$ \\
\hline PRE TEST - GC & 4,095238095 & 1,19 \\
\hline POST TEST - GC & 4,523809524 & 1,05 \\
\hline $\begin{array}{c}\text { PROMEDIO } \\
\text { GENERAL } \\
\text { Grupo de control }\end{array}$ & $\mathbf{4 , 3 0 9 5 2 3 8 1}$ & $\mathbf{1 , 1 2 1 6 8 3 6 6 8}$ \\
\hline PRE TEST - GE & 3,61 & 1,97 \\
\hline POST TEST-GE & 7,30 & 1,63 \\
\hline $\begin{array}{c}\text { PROMEDIO } \\
\text { GENERAL } \\
\text { Grupo experimental }\end{array}$ & $\mathbf{5 , 4 5 6 5 2 1 7 3 9}$ & $\mathbf{1 , 7 9 9 6 0 3 4 5 1}$ \\
\hline
\end{tabular}

Fuente: Microsoft Excel

Elaborado por: Armas Paul, 2017

Tabla 13. Análisis comparativo

\begin{tabular}{|c|c|c|c|}
\hline GRUPO & $\mathbf{N}$ & Promedio & DESVIACIÓN ESTÁNDAR(S) \\
\hline Control & 21 & 4,3095 & 1.12 \\
\hline Experimental & 23 & 5,4565 & 1,63 \\
\hline
\end{tabular}

Elaborado por: Armas Paul, 2017

\section{Determinación de valores críticos y zonas de rechazo}

Mediante el cálculo de la prueba paramétrica $\mathrm{Z}$ se rechaza la hipótesis nula si:

$$
Z_{c}<-Z_{T} \quad Z_{c}<=-1,96
$$

$O$ también $Z_{c}>Z_{T} Z_{c}>=1,96$ 
Donde $\mathbf{Z}_{\mathbf{T}}$, es el valor teórico de $\mathrm{Z}$ para un nivel de significación del $5 \%, \alpha=0,05$; es decir que la investigación tendrá un $95 \%$ de confiabilidad; caso contrario se acepta la hipótesis de investigación con una de las dos alternativas.

Los datos son:

$$
\begin{array}{ccc}
\overline{x_{B}}=5,46 \overline{x_{c}}=4,31 & \sigma_{\mathrm{e}}=1,63 \quad \sigma_{\mathrm{c}}=1,12 n_{\mathrm{e}}=21 \quad n_{\mathrm{c}}=23 \\
Z=\frac{\overline{x_{e}}-\overline{x_{c}}}{\sqrt{\frac{\sigma_{e}{ }^{2}}{n_{e}}+\frac{\sigma_{c}^{2}}{n_{c}}}} & \Rightarrow Z_{c}=2,56
\end{array}
$$

\section{Toma de decisión estadística}

Comparando $\mathrm{Z}$ calculado con $\mathrm{Z}$ teórico tenemos:

$$
\mathbf{Z}_{\mathbf{c}}>\mathbf{Z}_{\mathbf{T}} \Rightarrow 2,56>1,96
$$

$\mathbf{Z}_{\mathbf{c}}=\mathbf{2 , 5 6}$ se encuentra en la zona de rechazo de la hipótesis nula, por lo que nos conlleva a rechazar la hipótesis nula $H_{0}: \bar{x}_{B}=\bar{x}_{C}$ y aceptar la hipótesis de investigación Hi: $\bar{x}_{B} \neq \bar{x}_{C}$ con la alternativa $\mathrm{A}_{1}: \bar{x}_{B}>\bar{x}_{C}$.

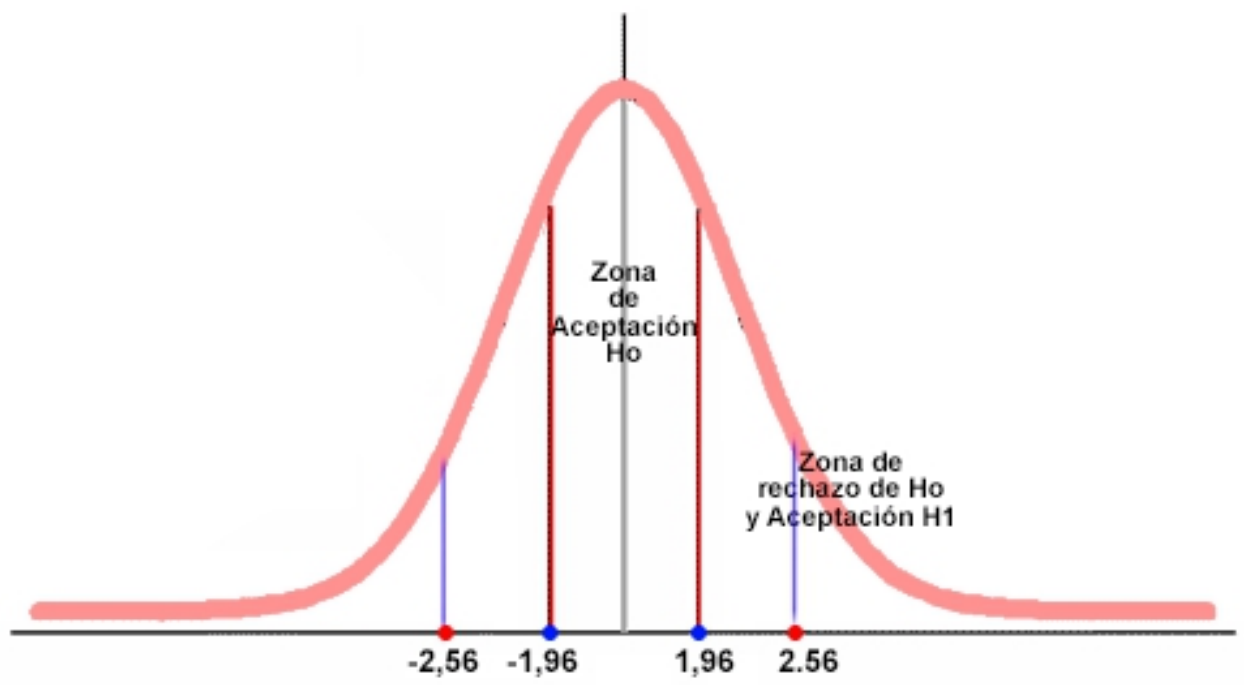

Gráfico 7. Valores de Z Teórico y Z Calculado

Con un nivel de significación de 0,05 equivalente al $+/-1,96$, se rechaza la hipótesis nula Ho ya que la solución $\mathrm{Zt}=2.56$ que se encuentra en el intervalo de la región de rechazo de Ho. En consecuencia, se acepta la hipótesis alternativa H1. Es decir que las actividades de la plataforma Duolingo incide en el desarrollo de las habilidades comunicacionales verbales 
del idioma inglés en los estudiantes de cuarto nivel del Centro de Idiomas de la Escuela Superior Politécnica de Chimborazo.

\section{CONCLUSION}

A través del proceso de la investigación, se evidenció la importancia del conocimiento que debe tener el docente sobre el manejo y uso de la plataforma Duolingo, pues su falta de capacitación podría modificar el plan de intervención y afectaría a las actividades para desarrollar las habilidades comunicacionales verbales del idioma inglés dentro de los tiempos previstos. De igual manera se evidencio que los estudiantes se adaptaron rápidamente y presentaron pocas barreras de accesibilidad, navegación y uso de la plataforma.

El modelo computacional uno a uno soportado por la plataforma Duolingo, permitió que el estudiante de forma autónoma repase los contenidos practicados en clase; permitiendo retroalimentación permanente enmarcados en los modelos pedagógicos conectivista y conceptual. Además, se observó que las actividades propuestas por la herramienta Duolingo se adapta a las etapas de desarrollo cognitivo de los estudiantes.

La herramienta Duolingo desde el punto de vista didáctico cumplió con las expectativas esperadas, puesto que permitió la integración de temas y conceptos para que los estudiantes se familiaricen con los descriptores y al mismo tiempo desarrollen las habilidades comunicativas del idioma inglés.

Los descriptores fueron elementos determinantes para el desarrollo de las habilidades comunicacionales verbales de inglés, ya que las actividades realizadas con el grupo experimental, se enfocaron para que incida de manera positiva; por lo que su aplicación a través del plan de intervención, permitió que las evaluaciones post test sean mejores que pre test del mismo grupo experimental, como también del grupo de control, al cual no se aplicó el plan de intervención.

Sin duda el grupo de control presento resultados inferiores al grupo experimental, cómo lo demostró la interpretación de las gráficas y el análisis estadístico, donde se evidenció que las medias y la desviación estándar promedio se impusieron y permitió afirmar que con el uso de la plataforma Duolingo se incidió en el desarrollo de las habilidades comunicativas verbales de inglés en los estudiantes del cuarto nivel del Centro de Idiomas de la ESPOCH.

\section{References:}

1. Alzate, D., (2015). "Evaluación de la herramienta Duolingo en el aprendizaje del inglés en niños de tercer grado”. Colombia.

2. Baddeley, A., D., (1990), Human Memory: Theory and Practice. Hove: Erlbaum Associates. 
3. Bower, G., H., (1970). "Analysis of a mnemonic device". American Science. Vol.58. 496-510.

4. Bransford, J., D., (1979), Human Cognition, Belmont, CA: Wadsworth.

5. Canale, M., Swain, M., (1980), Theoretical Bases of Communicative Approaches to Second Language Teaching and Testing. Recuperado de https://segue.atlas.uiuc.edu/uploads/nppm/CanaleSwain.80.pdf

6. Díaz B., J., Martins, A., (1986), Estrategia de Enseñanza-Aprendizaje. San José, Costa Rica: IICA.

7. Gavarri, S., L., (2016). El aprendizaje de lenguas extranjeras mediado por las TIC: Aprender inglés con Duolingo. El Toldo de Astier, 7 (12), 56-65. En Memoria Académica. Disponible en: http://www.memoria.fahce.unlp.edu.ar/art_revistas/pr.7199/pr.7199.p df

8. Navarro, L., Buenos Aires. Sede Regional, U.-I. (2004). La Escuela y las condiciones sociales para aprender y enseñar: equidad social y educación en sectores de pobreza urbana (Chile). http://lst-iiep.iiepunesco.org/cgi-

bin/wwwi32.exe/[in=epidoc1.in]/?t2000=022457/(100).

9. Ruiz, Y., (2008). 25 años de lingüística aplicada en España: Hitos y Retos, ASESLA, España, https://www.um.es/lacell/aesla/contenido/pdf/3/ruiz.pdf

10. Vesselinov R., y Grego, J., (2012), Duolingo Effectiveness Study. City University of NewYork. USA. 